\title{
MULTIVARIATE HARDY-TYPE INEQUALITIES
}

\author{
Z̆. HANJS̆, C. E. M. PEARCE AND J. PEC̆ARIĆ
}

\begin{abstract}
We present transparent proofs for some multivariate versions of both continuous and discrete Hardy-type inequalities. Our theorems subsume several known results and simplify the proofs of existing results.
\end{abstract}

\section{Introduction}

In 1920, Hardy [2] proved the following theorem.

Theorem A. If $p>1, f(x) \geq 0$ for $0<x<\infty$ and $G(x)=x^{-1} \int_{0}^{x} f(t) d t$, then

$$
\int_{0}^{\infty} G^{p}(x) d x<\left(\frac{p}{p-1}\right)^{p} \int_{0}^{\infty} f^{p}(x) d x
$$

unless $f \equiv 0$. The multiplicative constant is best-possible.

In the same article, Hardy also established a discretized form of this result.

Theorem B. If $p>1, a_{n} \geq 0$ and $A_{n}=\sum_{i=1}^{n} a_{i}(n \geq 1)$, then

$$
\sum_{n=1}^{\infty}\left(\frac{A_{n}}{n}\right)^{p}<\left(\frac{p}{p-1}\right)^{p} \sum_{n=1}^{\infty} a_{n}^{p}
$$

unless each $a_{n}=0$. The constant is best-possible.

In the case of Theorem B, he was unable to fix the multiplicative constant. This was rectified by Landau [6].

These relations have since been the object of sustained study and have undergone extensive generalization and found numerous applications. For comprehensive reviews of developments see the monograph of Opic and Kufner [9] and Chapter 4 of the monograph of Mitrinović, Pečarić and Fink [8]. An important early contribution was made by Copson [1], who established the following generalization of Theorem B.

Received May 27, 1999.

2000 Mathematics Subject Classification. Primary 26D15; secondary 26D20.

Key words and phrases. Hardy's inequality, multivariable generalization. 
Theorem C. Suppose $p>1, \lambda_{n}>0, \Lambda_{n}=\sum_{i=1}^{n} \lambda_{i}, a_{n}>0$ and $A_{n}=\sum_{i=1}^{n} \lambda_{i} a_{i}$ $(n \geq 1)$. If $\sum_{n=1}^{\infty} \lambda_{n} a_{n}^{p}$ converges, then

$$
\sum_{n=1}^{\infty} \lambda_{n}\left(\frac{A_{n}}{\Lambda_{n}}\right)^{p}<\left(\frac{p}{p-1}\right)^{p} \sum_{n=1}^{\infty} \lambda_{n} a_{n}^{p} .
$$

The constant is best-possible.

This reduces to Theorem $\mathrm{B}$ when the constants $\lambda_{n}$ assume a common value. In 1928 Hardy [3] provided a generalization of Theorem A.

Theorem D. Suppose $p>1, m \neq 1, f(x) \geq 0$ and $F(x)$ is defined by

$$
F(x)= \begin{cases}\int_{0}^{x} f(t) d t, & m>1 \\ \int_{x}^{\infty} f(t) d t, & m<1\end{cases}
$$

Then

$$
\int_{0}^{\infty} x^{-m} F^{p}(x) d x<\left\{\frac{p}{|m-1|}\right\}^{p} \int_{0}^{\infty} x^{p-m} f^{p}(x) d x
$$

unless $f \equiv 0$. The constant is best-possible.

This reduces to Theorem A for $m=p$.

A recent extension of Hardy's discrete inequality in the direction given by Copson in Theorem $\mathrm{C}$ is the following result of Hwang and Yang [5].

Theorem E. Suppose $p>1, \beta_{n}>0, \lambda_{n}>0, a_{n}>0(n \geq 1)$ and that $\sum_{n=1}^{\infty} \lambda_{n} a_{n}^{p}$ converges. Define $\Lambda_{n}=\sum_{i=1}^{n} \beta_{i} \lambda_{i}$ and $A_{n}=\sum_{i=1}^{n} \beta_{i} \lambda_{i} a_{i}(n \geq 1)$. If there exists $K>0$ such that

$$
p-1+\frac{\left(\beta_{n+1}-\beta_{n}\right) \Lambda_{n}}{\beta_{n+1} \beta_{n} \lambda_{n}} \geq \frac{p}{K} \quad \text { for } n=1,2,3, \ldots,
$$

then

$$
\sum_{n=1}^{\infty} \lambda_{n}\left(\frac{A_{n}}{\Lambda_{n}}\right)^{p} \leq K^{p} \sum_{n=1}^{\infty} \lambda_{n} a_{n}^{p}
$$

This has led to generalizations by Hwang [4] to discrete inequalities of Hardy type involving multiple variables. For earlier work on discrete Hardy-type inequalities see Pachpatte [10]. The proofs involved are relatively complicated. Yang and Jean [14] and Pachpatte [13] have given several two-variable versions and Pachpatte [11-12] multivariate versions of the continuous Hardy inequality, again via fairly involved proofs.

Our aim in this paper is to provide transparent treatments of multivariate inequalities of Hardy type both for the discrete and for the continuous case. In Section 2 we show that several results for the continuous case may be derived directly from known univariate results obtained by Lee and Yang [4]. Our results subsume those of [11] and [14]. In section 3 we give a parallel analysis for the discrete case. Our results generalize [10, Theorem 1] and provide a simpler proof for the main result of [4]. 


\section{Continuous Multivariate Inequalities}

Our derivation is based on the following results derived by Lee and Yang.

Theorem 2.1. Suppose $p>1$ and $f(x) \geq 0$ and that $r(x) \geq 0$ is absolutely continuous for $x>0$. Define $R(x)=\int_{0}^{x} r(t) d t$. If $m>1$ and there is $a>0$ such that

$$
p-1+\frac{R(x) r^{\prime}(x)}{r^{2}(x)}-\frac{(p-m) R(x)}{x r(x)} \geq \frac{p}{\lambda}
$$

for almost all $x>0$, then

$$
\int_{0}^{\infty} x^{p-m}\left(\frac{1}{R(x)} \int_{0}^{x} r(s) f(s) d s\right)^{p} d x \leq \lambda^{p} \int_{0}^{\infty} x^{p-m} f^{p}(x) d x .
$$

Theorem 2.2. Let $p, f, r$ be as in Theorem 2.1 and define $G(x)=\frac{1}{x r(x)} \int_{0}^{x} r(t) f(t) d t$. If $m>1$ and there is a $\lambda>0$ such that

$$
\frac{m-1}{p}+\frac{x r^{\prime}(x)}{r(x)} \geq \frac{1}{\lambda}
$$

for almost all $x>0$, then

$$
\int_{0}^{\infty} x^{p-m} G^{p}(x) d x \leq \lambda^{p} \int_{0}^{\infty} x^{p-m} f^{p}(x) d x .
$$

Theorem 2.3. Let $p, f, r$ be as in Theorem 2.1, $\alpha>1$ and $R(x)=\int_{x}^{\infty} r(t) d t$. If $m<1$ and there is a $\lambda>0$ such that

$$
p-1-\frac{(m+\alpha p-p) R(x)}{x r(x)}-\frac{R(x) r^{\prime}(x)}{r^{2}(x)} \geq \frac{p}{\lambda}
$$

for almost all $x \geq 0$, then

$$
\int_{0}^{\infty} x^{p-\alpha p-m}\left(\frac{1}{R(x)} \int_{x}^{\infty} t^{\alpha} r(t) f(t) d t\right)^{p} d x \leq \lambda^{p} \int_{0}^{\infty} x^{p-m} f^{p}(x) d x .
$$

Theorem 2.4. Let $p, \alpha, f, r$ be as in Theorem 2.3 and $G(x)=\int_{x}^{\infty} t^{\alpha} r(t) d t /\left\{x^{\alpha} r(x)\right\}$. If $m<1$ and there is a $\lambda>0$ such that

$$
\frac{1-m}{p}-\frac{x r^{\prime}(x)}{r(x)}-\alpha \geq \frac{1}{\lambda}
$$

for almost all $x>0$, then

$$
\int_{0}^{\infty} x^{-m} G^{p}(x) d x \leq \lambda^{p} \int_{0}^{\infty} x^{p-m} f^{p}(x) d x .
$$


We now proceed to multivariate versions of these four theorems. First we consider a generalization of Theorem 2.1. This subsumes the main theorem of Pachpatte [11] under the case $m=p$.

Theorem 2.5. Let $p>1$ and $f$ be a nonnegative integrable function defined on the positive orthant $\left\{\left(x_{1}, \ldots, x_{n}\right): x_{i}>0, i=1, \ldots, n\right\}$. Suppose $r_{i}(i=1, \ldots, n)$ is positive and absolutely continuous on $(0, \infty)$ and define $R_{i}\left(x_{i}\right)=\int_{0}^{x_{i}} r_{i}(t) d t$. If $m_{i}>1$ and there exist constants $\lambda_{i}>0(i=1, \ldots, n)$ such that

$$
p-1+\frac{R_{i}\left(x_{i}\right) r_{i}^{\prime}\left(x_{i}\right)}{r_{i}^{2}\left(x_{i}\right)}-\frac{\left(p-m_{i}\right) R_{i}\left(x_{i}\right)}{x_{i} r_{i}\left(x_{i}\right)} \geq \frac{p}{\lambda_{i}}
$$

for all $x_{i} \in(0, \infty)(i=1, \ldots, n)$, then

$$
\begin{aligned}
& \int_{0}^{\infty} \cdots \int_{0}^{\infty}\left(\prod_{i=1}^{n} x_{i}^{-m_{i}}\right) H^{p}\left(x_{1}, \ldots, x_{n}\right) d x_{1} \cdots d x_{n} \\
\leq & \left(\prod_{i=1}^{n} \lambda_{i}\right)^{p} \int_{0}^{\infty} \cdots \int_{0}^{\infty}\left(\prod_{i=1}^{n} x_{i}^{p-m_{i}}\right) f^{p}\left(x_{1}, \ldots, x_{n}\right) d x_{1} \cdots d x_{n},
\end{aligned}
$$

where

$$
H\left(x_{1}, \ldots, x_{n}\right)=\int_{0}^{x_{1}} \cdots \int_{0}^{x_{n}}\left\{\prod_{i=1}^{n} \frac{r_{i}\left(s_{i}\right) x_{i}}{R_{i}\left(x_{i}\right)}\right\} f\left(s_{1}, \ldots, s_{n}\right) d s_{1} \cdots d s_{n}
$$

Proof. We proceed via mathematical induction. First note that the standing condition (2.3) for $n=k+1$ subsumes that for $n=k$. A basis is provided by Theorem 2.1, which provides the result for $n=1$. For the inductive step, suppose (2.4) holds for $n=k$ and conditions (2.3) for $n=k+1$. Since the integrand is nonnegative, Fubini's theorem applies and we may arrange the left-hand side of (2.4) for $n=k+1$ as

$$
\begin{aligned}
& \int_{0}^{\infty} \frac{x_{k+1}^{p-m_{k+1}}}{R_{k+1}^{p}\left(x_{k+1}\right)}\left\{\int_{0}^{\infty} \cdots \int_{0}^{\infty}\left(\prod_{i=1}^{k} x_{i}^{-m_{i}}\right)\right. \\
& \left.\times\left[\int_{0}^{x_{1}} \cdots \int_{0}^{x_{k}}\left\{\prod_{i=1}^{k} \frac{x_{i} r_{i}\left(s_{i}\right)}{R_{i}\left(x_{i}\right)}\right\} F\left(s_{1}, \ldots, s_{k}\right) d s_{1} \cdots d s_{k}\right]^{p} d x_{1} \cdots d x_{k}\right\} d x_{k+1},
\end{aligned}
$$

where $x_{k+1}$ is regarded as a parameter in the function

$$
F\left(s_{1}, \ldots, s_{k}\right):=\int_{0}^{x_{k+1}} r_{k+1}\left(s_{k+1}\right) f\left(s_{1}, \ldots, s_{k}, s_{k+1}\right) d s_{k+1} .
$$

By the inductive hypothesis, the expression inside the outer braces in (2.5) is bounded above by

$$
\left(\prod_{i=1}^{k} \lambda_{i}^{p}\right) \int_{0}^{\infty} \cdots \int_{0}^{\infty}\left(\prod_{i=1}^{k} x_{i}^{p-m_{i}}\right) F^{p}\left(x_{1}, \ldots, x_{k}\right) d x_{1} \cdots d x_{k}
$$


and so the left-hand side of (2.4) for $n=k+1$ is bounded above by

$$
\begin{aligned}
& \left(\prod_{i=1}^{k} \lambda_{i}^{p}\right) \int_{0}^{\infty} \cdots \int_{0}^{\infty}\left(\prod_{i=1}^{k} x_{i}^{p-m_{i}}\right) \int_{0}^{\infty} x_{k+1}^{p-m_{k+1}}\left\{\frac{1}{R_{k+1}\left(x_{k+1}\right)}\right. \\
& \left.\times \int_{0}^{x_{k+1}} r_{k+1}\left(s_{k+1}\right) f\left(x_{1}, \ldots, x_{k}, s_{k+1}\right) d s_{k+1}\right\}^{p} d x_{k+1} d x_{1} \cdots d x_{k} .
\end{aligned}
$$

By Theorem 2.1, this in turn is bounded above by

$$
\begin{aligned}
& \left(\prod_{i=1}^{k} \lambda_{i}\right)^{p} \int_{0}^{\infty} \cdots \int_{0}^{\infty}\left(\prod_{i=1}^{k} x_{i}^{p-m_{i}}\right) \lambda_{k+1}^{p} \int_{0}^{\infty} x_{k+1}^{p-m_{k+1}} f^{p}\left(x_{1}, \ldots, x_{k}, x_{k+1}\right) d x_{k+1} d x_{1} \cdots d x_{k} \\
= & \left(\prod_{i=1}^{k+1} \lambda_{i}\right)^{p} \int_{0}^{\infty} \cdots \int_{0}^{\infty}\left(\prod_{i=1}^{k+1} x_{i}^{p-m_{i}}\right) f^{p}\left(x_{1}, \ldots, x_{k+1}\right) d x_{1} \cdots d x_{k+1},
\end{aligned}
$$

and the inductive step is established.

Exactly similar reasoning may be used to establish the three following theorems from Theorems 2.2, 2.3 and 2.4 .

Theorem 2.6. Let $p, f, r_{i}(i=1, \ldots, n)$ be as in Theorem 2.5. If $m_{i}>1$ and there exist $\lambda_{i}>0(i=1, \ldots, n)$ such that

$$
\frac{m_{i}-1}{p}+\frac{x_{i} r_{i}^{\prime}\left(x_{i}\right)}{r_{i}\left(x_{i}\right)} \geq \frac{1}{\lambda_{i}}, \text { for all } x_{i} \in(0, \infty)(i=1, \ldots, n),
$$

then

$$
\begin{aligned}
& \int_{0}^{\infty} \cdots \int_{0}^{\infty}\left(\prod_{i=1}^{n} x_{i}^{-m_{i}}\right) H^{p}\left(x_{1}, \ldots, x_{n}\right) d x_{1} \cdots d x_{n} \\
\leq & \left(\prod_{i=1}^{n} \lambda_{i}\right)^{p} \int_{0}^{\infty} \cdots \int_{0}^{\infty}\left(\prod_{i=1}^{n} x_{i}^{p-m_{i}}\right) f^{p}\left(x_{1}, \ldots, x_{n}\right) d x_{1} \cdots d x_{n}
\end{aligned}
$$

where

$$
H\left(x_{1}, \ldots, x_{n}\right)=\int_{0}^{x_{1}} \cdots \int_{0}^{x_{n}}\left(\prod_{i=1}^{n} \frac{r_{i}\left(s_{i}\right)}{r_{i}\left(x_{i}\right)}\right) f\left(s_{1}, \ldots, s_{n}\right) d s_{1} \cdots d s_{n}
$$

Theorem 2.7. Let $p, f, r_{i}(i=1, \ldots, n)$ be as in Theorem $2.5, \alpha_{i}>1$ and $R_{i}\left(x_{i}\right)=$ $\int_{x_{i}}^{\infty} r_{i}\left(s_{i}\right) d s_{i}(i=1, \ldots, n)$. Suppose further that $m_{i}<1$ and there exist constants $\lambda_{i}$ $(i=1, \ldots, n)$ such that

$$
p-1-\frac{\left(m_{1}+\alpha_{i} p-p\right) R_{i}\left(x_{i}\right)}{x_{i} r_{i}\left(x_{i}\right)}-\frac{R_{i}\left(x_{i}\right) r_{i}^{\prime}\left(x_{i}\right)}{r_{i}^{2}\left(x_{i}\right)} \geq \frac{p}{\lambda_{i}}
$$


for all $x_{i} \in(0, \infty)(i=1, \ldots, n)$. Then

$$
\begin{aligned}
& \int_{0}^{\infty} \cdots \int_{0}^{\infty}\left(\prod_{i=1}^{n} x_{i}^{-m_{i}}\right) H^{p}\left(x_{1}, \ldots, x_{n}\right) d x_{1} \cdots d x_{n} \\
\leq & \left(\prod_{i=1}^{n} \lambda_{i}\right)^{p} \int_{0}^{\infty} \cdots \int_{0}^{\infty}\left(\prod_{i=1}^{n} x_{i}^{p-m_{i}}\right) f^{p}\left(x_{1}, \ldots, x_{n}\right) d x_{1} \cdots d x_{n},
\end{aligned}
$$

where

$$
H\left(x_{1}, \ldots, x_{n}\right)=\int_{x_{1}}^{\infty} \cdots \int_{x_{n}}^{\infty}\left(\prod_{i=1}^{n} \frac{x_{i}^{1-\alpha_{i}} s_{i}^{\alpha_{i}} r_{i}\left(s_{i}\right)}{R_{i}\left(x_{i}\right)}\right) f\left(s_{1}, \ldots, s_{n}\right) d s_{1} \cdots d s_{n} .
$$

Theorem 2.8. Let $p, \alpha_{i}, f, r_{i}(i=1, \ldots, n)$ be as in Theorem 2.7. Suppose $m_{i}<1$ and there exist $\lambda_{i}(i=1, \ldots, n)$ such that

$$
\frac{1-m_{i}}{p}-\frac{x_{i} r_{i}^{\prime}\left(x_{i}\right)}{r_{i}\left(x_{i}\right)}-\alpha_{i} \geq \frac{1}{\lambda_{i}}, \quad \text { for all } x_{i} \in(0, \infty)(i=1, \ldots, n) .
$$

Then

$$
\begin{aligned}
& \int_{0}^{\infty} \cdots \int_{0}^{\infty}\left(\prod_{i=1}^{n} x_{i}^{-m_{i}}\right) H^{p}\left(x_{1}, \ldots, x_{n}\right) d x_{1} \cdots d x_{n} \\
\leq & \left(\prod_{i=1}^{n} \lambda_{i}\right)^{p} \int_{0}^{\infty} \cdots \int_{0}^{\infty}\left(\prod_{i=1}^{n} x_{i}^{p-m_{i}}\right) f^{p}\left(x_{1}, \ldots, x_{n}\right) d x_{1} \cdots d x_{n},
\end{aligned}
$$

where

$$
H\left(x_{1}, \ldots, x_{n}\right)=\int_{x_{1}}^{\infty} \cdots \int_{x_{n}}^{\infty}\left(\prod_{i=1}^{n} \frac{s_{i}^{\alpha_{i}} r_{i}\left(s_{i}\right)}{x_{i}^{\alpha_{i}} r_{i}\left(x_{i}\right)}\right) f\left(s_{1}, \ldots, s_{n}\right) d s_{1} \cdots d s_{n} .
$$

For $n=2$, Theorems 2.5-2.8 extend the two-variable results of Yang and Jean [14].

\section{Discrete Multivariate Inequalities}

For use in the sequel we define $B_{n} \subset \mathbf{Z}_{+}^{n}$ by

$$
B_{n}:=\left\{x=\left(x_{1}, \ldots, x_{n}\right): x_{i} \in Z_{+}\right\} .
$$

Similarly for $x \in B_{n}$ we define

$$
C_{n}(x):=\left\{y=\left(y_{1}, \ldots, y_{n}\right): y \in B_{n}, y_{i} \leq x_{i}(i=1, \ldots, n)\right\} .
$$

For a function $u: B_{n} \rightarrow \mathbf{R}$ it is convenient to introduce the shorthand

$$
\sum_{B_{n}} u(y):=\sum_{y_{1}=1}^{\infty} \cdots \sum_{y_{n}=1}^{\infty} u\left(y_{1}, y_{2}, \ldots, y_{n}\right)
$$


and

$$
\sum_{C_{n}(x)} u(y):=\sum_{y_{1}=1}^{x_{1}} \cdots \sum_{y_{n}=1}^{x_{n}} u\left(y_{1}, y_{2}, \ldots, y_{n}\right) .
$$

We are now in a position to state a discrete multivariate Hardy-type inequality given by Pachpatte [10].

Theorem 3.1. Suppose $p>0$ is a constant, $f(x) \geq 0$ for $x \in B_{n}$ and $A_{n}(x)=$ $\sum_{C_{n}(x)} f(x)$ for $x \in B_{n}$. Then

$$
\sum_{B_{n}}\left\{\frac{A_{n}(x)}{\prod_{i=1}^{n} x_{i}}\right\}^{p} \leq\left(\frac{p}{p-1}\right)^{n p} \sum_{B_{n}} f^{p}(x) .
$$

Equality holds if $f(x)=0$ for all $x_{i}(i=1,2, \ldots, n)$.

We provide a simple proof of the following extension of Theorem C. Theorem 3.1 occurs as the special case $\lambda_{i}\left(x_{i}\right)=1$.

Theorem 3.2. Let $p>1, a(x)>0$ and $\lambda_{i}\left(x_{i}\right)>0$ for $x_{i} \geq 1(i=1,2, \ldots, n)$. We define $\Lambda_{i}(x)=\sum_{y_{i}=1}^{x_{i}} \lambda_{i}\left(y_{i}\right)$ for $i=1,2, \ldots, n$ and

$$
A_{n}(x)=\sum_{C_{n}(x)}\left\{\prod_{i=1}^{n} \lambda_{i}\left(y_{i}\right)\right\} a(y) \quad x \in B_{n} .
$$

Suppose $\sum_{B_{n}} \prod_{i=1}^{n} \lambda_{i}\left(x_{i}\right) a^{p}(x)$ converges for $x \in B_{n}$. Then

$$
\sum_{B_{n}}\left(\prod_{i=1}^{n} \lambda_{i}\left(x_{i}\right)\right)\left\{\frac{A_{n}(x)}{\prod_{i=1}^{n} \Lambda_{i}\left(x_{i}\right)}\right\} \leq\left(\frac{p}{p-1}\right)^{n p} \sum_{B_{n}}\left(\prod_{i=1}^{n} \lambda_{i}\left(x_{i}\right)\right) a^{p}(x) .
$$

Proof. We employ induction. Theorem $\mathrm{C}$ gives the result for $n=1$, supplying a basis. Let (3.1) be true for $n=k$, that is, let

$$
\sum_{B_{k}}\left(\prod_{i=1}^{k} \lambda_{i}\left(x_{i}\right)\right)\left\{\frac{A_{k}(x)}{\prod_{i=1}^{k} \Lambda_{i}\left(x_{i}\right)}\right\}^{p} \leq\left(\frac{p}{p-1}\right)^{k p} \sum_{B_{k}}\left(\prod_{i=1}^{k} \lambda_{i}\left(x_{i}\right)\right) a^{p}(x) .
$$

For $n=k+1$, the left-hand side of (3.1) is

$$
\sum_{B_{k}}\left(\prod_{i=1}^{k} \lambda_{i}\left(x_{i}\right)\right) \sum_{x_{k+1}=1}^{\infty} \lambda_{k+1}\left(x_{k+1}\right)\left\{\frac{A_{k+1}(x)}{\Lambda_{k+1}\left(x_{k+1}\right) \prod_{i=1}^{k} \Lambda_{i}\left(x_{i}\right)}\right\}^{p}
$$




$$
=\sum_{B_{k}}\left(\prod_{i=1}^{k} \frac{\lambda_{i}\left(x_{i}\right)}{\left(\Lambda_{i}\left(x_{i}\right)\right)^{p}}\right) \sum_{x_{k+1}=1}^{\infty} \lambda_{k+1}\left(x_{k+1}\right)\left\{\frac{\sum_{y_{k+1}=1}^{x_{k+1}} \lambda_{k+1}\left(y_{k+1}\right) \sum_{C_{k}(x)}\left(\prod_{i=1}^{k} \lambda_{i}\left(y_{i}\right)\right) a(y)}{\Lambda_{k+1}\left(x_{k+1}\right)}\right\}^{p} .
$$

By Theorem C, the expression in braces is dominated by

$$
\left(\frac{p}{p-1}\right)^{p}\left\{\sum_{C_{k}(x)}\left(\prod_{i=1}^{k} \lambda_{i}\left(y_{i}\right)\right) a\left(y_{1}, \ldots, y_{k}, x_{k+1}\right)\right\}^{p} .
$$

Hence the left-hand side of (3.1) is dominated by

$$
\left(\frac{p}{p-1}\right)^{p} \sum_{x_{k+1}=1}^{\infty} \lambda_{k+1}\left(x_{k+1}\right) \sum_{B_{k}}\left(\prod_{i=1}^{k} \lambda_{i}\left(x_{i}\right)\right)\left\{\frac{\sum_{C_{k}(x)}\left(\prod_{i=1}^{k} \lambda_{i}\left(y_{i}\right)\right) a\left(y_{1}, \ldots, y_{k}, x_{k+1}\right)}{\prod_{i=1}^{k} \Lambda_{i}\left(x_{i}\right)}\right\}^{p} .
$$

By the inductive hypothesis (3.2), this in turn is dominated by

$$
\begin{aligned}
& \left(\frac{p}{p-1}\right)^{p} \sum_{x_{k+1}=1}^{\infty} \lambda_{k+1}\left(x_{k+1}\right)\left(\frac{p}{p-1}\right)^{k p} \sum_{B_{k}}\left(\prod_{i=1}^{k} \lambda_{i}\left(x_{i}\right)\right) a^{p}\left(x_{1}, \ldots, x_{k}, x_{k+1}\right) \\
= & \left(\frac{p}{p-1}\right)^{(k+1)_{p}} \sum_{B_{k+1}}\left(\prod_{i=1}^{k+1} \lambda_{i}\left(x_{i}\right)\right) a^{p}\left(x_{1}, \ldots, x_{k+1}\right) .
\end{aligned}
$$

Hence we have the desired result for $n=k+1$, as required.

To conclude, we give a simple proof of the following result of Hwang [4], which generalizes Theorem E.

Theorem 3.3. Let $p>1, a(x)>0, \beta_{i}\left(x_{i}\right)>0, \lambda_{i}\left(x_{i}\right)>0$ for $x_{i} \geq 1(i=$ $1,2, \ldots, n)$. Define $\Lambda_{i}(x)=\sum_{y_{i}=1}^{x_{i}} \beta_{i}\left(y_{i}\right) \lambda_{i}\left(y_{i}\right)$ for $i=1,2, \ldots, n$ and $A(x)=\sum_{C_{n}(x)}$ $\left\{\prod_{i=1}^{n} \beta_{i}\left(y_{i}\right) \lambda_{i}\left(y_{i}\right)\right\} a(y)$ for $x \in B_{n}$. Further, suppose $\sum_{B_{n}} \prod_{i=1}^{n} \lambda_{i}\left(x_{i}\right) a^{p}(x)$ converges for $x \in B_{n}$. If there exist constants $K_{i}>0$ such that

$$
p-1+\frac{\left[\beta_{i}\left(x_{i}+1\right)-\beta_{i}\left(x_{i}\right)\right] \Lambda_{i}\left(x_{i}\right)}{\beta_{i}\left(x_{i}+1\right) \beta_{i}\left(x_{i}\right) \lambda_{i}\left(x_{i}\right)} \geq \frac{p}{K_{i}}
$$

for $x_{i} \geq 1(i=1,2, \ldots, n)$, then

$$
\sum_{B_{n}}\left(\prod_{i=1}^{n} \lambda_{i}\left(x_{i}\right)\right)\left\{\frac{A(x)}{\prod_{i=1}^{n} \Lambda_{i}\left(x_{i}\right)}\right\}^{p} \leq\left(\prod_{i=1}^{n} K_{i}\right)^{p} \sum_{B_{n}}\left(\prod_{i=1}^{n} \lambda_{i}\left(x_{i}\right)\right) a^{p}(x) .
$$


Equality holds in (3.5) if $a(x)=0$ for all $x_{i}(i=1,2, \ldots, n)$.

Proof. We give a proof by induction. A basis is supplied for $n=1$ by Theorem E. For the inductive step, suppose the theorem to be true for $n=k$ while the conditions hold for $n=k+1$.

For $n=k+1$, the left-hand side of (3.5) can be written as

$$
\sum_{B_{k}}\left(\prod_{i=1}^{k} \frac{\lambda_{i}\left(x_{i}\right)}{\Lambda_{i}^{p}\left(x_{i}\right)}\right) \sum_{x_{k+1}=1}^{\infty} \lambda_{k+1}\left(x_{k+1}\right)\left\{\frac{\sum_{y_{k+1}=1}^{x_{k+1}} \beta_{k+1}\left(y_{k+1}\right) \sum_{C_{k}(x)}\left(\prod_{i=1}^{k} \beta_{i}\left(y_{i}\right) \lambda_{i}\left(y_{i}\right)\right) a(y)}{\Lambda_{k+1}\left(x_{k+1}\right)}\right\}^{p} .
$$

By Theorem E, the expression in braces is less than or equal to

$$
\sum_{B_{k}} K_{k+1}^{p}\left\{\sum_{C_{k}(x)}\left(\prod_{i=1}^{k} \beta_{i}\left(y_{i}\right) \lambda_{i}\left(y_{i}\right)\right) a\left(y_{1}, \ldots, y_{k}, x_{k+1}\right)\right\}^{p} .
$$

Hence the left-hand side of (3.5) is less than or equal to

$$
K_{k+1}^{p} \sum_{x_{k+1}=1}^{\infty} \lambda_{k+1}\left(x_{k+1}\right) \sum_{B_{k}}\left(\prod_{i=1}^{k} \lambda_{i}\left(x_{i}\right)\right)\left\{\frac{\sum_{C_{k}(x)}\left(\prod_{i=1}^{k} \beta_{i}\left(y_{i}\right) \lambda_{i}\left(y_{i}\right)\right) a\left(y_{1}, \ldots, y_{k}, x_{k+1}\right)}{\prod_{i=1}^{k} \Lambda_{i}\left(x_{i}\right)}\right\}^{p} .
$$

By the inductive hypothesis, this is less than or equal to

$$
\begin{aligned}
& K_{k+1}^{p} \sum_{x_{k+1}=1}^{\infty} \lambda_{k+1}\left(x_{k+1}\right)\left(\prod_{i=1}^{k} K_{i}\right)^{p} \sum_{B_{k}}\left(\prod_{i=1}^{k} \lambda_{i}\left(x_{i}\right)\right) a^{p}\left(x_{1}, \ldots, x_{k}, x_{k+1}\right) \\
= & \left(\prod_{i=1}^{k+1} K_{i}\right)^{p} \sum_{B_{k+1}}\left(\prod_{i=1}^{k+1} \lambda_{i}\left(x_{i}\right)\right) a^{p}\left(x_{1}, \ldots, x_{k+1}\right)
\end{aligned}
$$

and the proof is complete.

\section{References}

[1] E. T. Copson, Note on a series of positive terms, J. London Math. Soc., 2(1927), 9-12.

[2] G. H. Hardy, Note on a theorem of Hilbert, Math. Zeitschr., 6(1920), 314-317.

[3] G. Hardy, Note on some points in the integral calculus, Messenger of Math., 57(1928), 12-16.

[4] D.-Y. Hwang, A many variable generalization of the discrete Hardy's inequality, Tamkang J. Math., 27(1996), 125-132. 
[5] D.-Y. Hwang and G.-S. Yang, Note on discrete Hardy's inequality, Tamkang J. Math., 21(1990), 333-336.

[6] E. Landau, A note on a theorem concerning series of positive terms, J. London Math. Soc., 1(1926), 38-39.

[7] K.-C. Lee and G.-S. Yang, On generalisation of Hardy inequality, Tamkang J. Math., 17 (1986), 109-119.

[8] D. S. Mitrinović, J. E. Pečarić and A. M. Fink, Inequalities involving functions and their integrals and derivatives, Kluwer, Dordrecht, 1991.

[9] B. Opic and A. Kufner, Hardy-type inequalities, Longman Scientific and Technical, Wiley, Harlow, Essex, New York, 1990.

[10] B. G. Pachpatte, A many variable generalisation of Hardy's inequality concerning a series of terms, Tamkang J. Math., 23(1992), 349-354.

[11] B. G. Pachpatte, On multivariate Hardy-type inequalities, Anal. Sti. Ale Univ. Al. I. Cusa Iasi, Matemat., 38(1992), 355-361.

[12] B. G. Pachpatte, On some generalizations of Hardy's integral inequality for functions of several variables, Demonstratio Math., 27(1994), 43-51.

[13] B. G. Pachpatte, On Hardy type integral inequalities for functions of two variables, Demonstratio Math., 28(1995), 239-244.

[14] G.-S. Yang and S.-P. Jean, Inequalities of Hardy type in two variables, Tamkang J. Math., 24(1993), 341-354.

Department of Mathematics, University of Zagreb, Zagreb, Croatia.

Department of Applied Mathematics, The University of Adelaide, Adelaide, SA 5005, Australia. 